\title{
Elliptic flow in nuclear collisions at the Large Hadron Collider
}

\author{
H. Niemi, K.J. Eskola and P.V. Ruuskanen* \\ Department of Physics, P.O.Box 35, FIN-40014 University of Jyväskylä, Finland \\ Helsinki Institute of Physics, P.O.Box 64, FIN-00014 University of Helsinki, Finland
}

(Dated: June 6, 2008)

\begin{abstract}
We use perfect-fluid hydrodynamical model to predict the elliptic flow coefficients in $\mathrm{Pb}+\mathrm{Pb}$ collisions at the Large Hadron Collider (LHC). The initial state for the hydrodynamical calculation for central $A+A$ collisions is obtained from the perturbative QCD + saturation (EKRT) model. The centrality dependence of the initial state is modeled by the optical Glauber model. We show that the baseline results obtained from the framework are in good agreement with the data from the Relativistic Heavy Ion Collider (RHIC), and show predictions for the $p_{T}$ spectra and elliptic flow of pions in $\mathrm{Pb}+\mathrm{Pb}$ collisions at the LHC. Also mass and multiplicity effects are discussed.
\end{abstract}

PACS numbers: 25.75.-q, 25.75.Dw, 25.75.Ld, 47.75.+f

\section{INTRODUCTION}

Azimuthal anisotropies of hadron spectra are a good measure of collective behavior of the dense particle system formed in the ultrarelativistic heavy ion collisions [1]. The origin of such anisotropies is thought to be the rescattering among the particles, which are initially produced in isotropic partonic interactions. These anisotropies can be quantified by the second Fourier coefficient $v_{2}$, the so called elliptic flow coefficient, of the azimuthal hadron distribution. In non-central $\mathrm{Au}+\mathrm{Au}$ collisions at the Relativistic Heavy Ion Collider (RHIC) at BNL the observed quite large $v_{2}$ is regarded as one of the strongest signals pointing towards the formation of thermalized strongly interacting partonic matter, the Quark-Gluon Plasma (QGP).

A common way to interpret the measured elliptic flow data is through full equilibrium, perfect-fluid hydrodynamical models, see e.g. reviews [2, 3, 4], with the Cooper-Frye freeze-out mechanism [5]. Consistency between these type of models and the measured data at RHIC is regarded as evidence for low viscosity [6] and fast thermalization [7] of the QGP. However, a complete description of the low- $p_{T}$ spectra and elliptic flow coefficients of pions simultaneously with e.g. those of protons is problematic in this simple approach.

To improve the modeling, several different approaches have been developed. If the chemical freeze-out is taken to happen before the complete kinetic freeze-out in the Cooper-Frye type of decoupling [8, 9], a good simultaneous description of e.g. the transverse momentum spectra of pions and protons can be obtained [10]. However, elliptic flow tends to be overestimated in these models. At the moment, perhaps the best agreement with the data is obtained from the hybrid models, which combine hydrodynamical treatment of the QGP and hadron cascade simulation of the hadronic interactions [11, 12, 13, 14]. It is clear that a proper understand- ing of hadronic interactions and the freeze-out mechanism are important in interpreting the observed data at RHIC. Also hydrodynamical codes with shear viscosity corrections have been recently developed for non-central collisions [15, 16, 17, 18, 19]. These calculations indicate also low values of viscosity in the expanding matter.

The key input to the hydrodynamical models are the initial state and the equation of state (EoS) of the QCD matter. When the initial state is obtained by fitting the hydrodynamical model to the RHIC data, the observed total hadron multiplicities fix the initial total entropy very well, but the transverse profiles of the initial densities are not as well constrained, see e.g. Ref. [17]. One possibility for controlling this uncertainty is to use theoretically predicted initial conditions. In this approach it is possible to predict the initial state for different collision energies and nuclei as well. This has been our strategy e.g. in Refs. 20, 21, 22, 23].

In [20] we used the initial state from the EKRT final state saturation model [22] and showed that the perturbative QCD + saturation + hydrodynamics approach gives a good description of the $p_{T}$ spectra of pions and kaons, and hadronic multiplicities in central $\mathrm{Au}+\mathrm{Au}$ collisions at RHIC. We also presented the predictions for the hadron $p_{T}$ spectra in central $\mathrm{Pb}+\mathrm{Pb}$ collisions at the Large Hadron Collider (LHC). We have also shown predictions for the elliptic flow at the LHC [24, 25]. The aim of the present work is to expand our previous studies of the hadron spectra and elliptic flow at the LHC and to probe the uncertainties in the predictions. A similar approach with a calculated initial state is used in Ref. [26], where the initial state is obtained from the Color-Glass Condensate model [27]. Other LHC predictions for the elliptic flow using the perfect-fluid hydrodynamics can be found in Refs. [25, 28, 29] and in the framework of viscous hydrodynamics in Ref. [30].

In this study we adopt the simple approach and use the perfect-fluid hydrodynamics for the space-time evolution of the matter, assuming full kinetic and chemical equilibrium throughout the evolution, see Sec. II. In spite of its restrictions discussed above, the full-equilibrium hy-

\footnotetext{
*Electronic address: harri.niemi, kari.eskola, vesa.ruuskanen@phys.jyu.forodynamical approach, once tested against the RHIC
} 
data, provides a good framework for baseline predictions of the low- $p_{T}$ pion spectra and elliptic flow coefficients at the LHC. As discussed in Sec. III below, the EKRT model gives us the initial state for the hydrodynamical evolution in central $A+A$ collisions for both RHIC and the LHC energies. Extension to non-central collisions is made by using the optical Glauber model at two different limits. The hydrodynamical evolution, elliptic flow, eccentricities and transverse flow at RHIC and LHC are discussed in Sec. IV. We will first, in Sec. V, show that the model results agree with the RHIC data, and then in Sec. VI present our predictions for the transverse momentum spectra of pions and for the elliptic flow coefficients of pions and protons at the LHC. Conclusions are given in Sec. VII.

\section{HYDRODYNAMICAL FRAMEWORK}

Once the initial energy density $\epsilon$ and the net-baryon density $n_{B}$ are given and the Equation of State (EoS) $P=P\left(\epsilon, n_{B}\right)$ is known, the evolution of matter can be described by relativistic hydrodynamics. By solving the perfect-fluid hydrodynamic equations, i.e. the local conservation of 4-momentum, $\partial_{\mu} T^{\mu \nu}=0$, and of netbaryon number, $\partial_{\mu}\left(n_{B} u^{\mu}\right)=0$, one obtains the spacetime evolution of all thermodynamic quantities and the collective flow velocity $u^{\mu}=\gamma\left(1, \mathbf{v}_{\mathbf{T}}, v_{z}\right)$ of the matter. When considering particle production at midrapidities, where the rapidity spectra are approximately flat, these equations can be simplified by assuming longitudinal boost invariance. In this case the longitudinal flow velocity is given by $v_{z}=z / t$ [31], and all hydrodynamical quantities become independent of the space-time rapidity $\eta=(1 / 2) \ln [(t+z) /(t-z)]$, i.e. they depend on the transverse coordinates $x$ and $y$, and the longitudinal proper time $\tau=\sqrt{t^{2}-z^{2}}$ only. This reduces the $(3+1)$ dimensional problem to a $(2+1)$-dimensional one. We write then the conservation laws in these variables and, including the EoS, solve them numerically by applying the SHASTA algorithm [32, 33.

In constructing the EoS 34], we describe the hightemperature phase as an ideal gas of massless quarks and gluons with number of flavors $N_{f}=3$ and a bag constant $B$, while the low-temperature phase is taken as an ideal gas of all hadronic states with $m<2 \mathrm{GeV}$ [35]. The QGP and hadron resonance gas (HRG) phases are connected via the Maxwell construction with mixed phase (MP) between the QGP and the HRG phase. The order of the phase transition has been shown to have only small effects on the hadron $p_{T}$ spectra and the elliptic flow of pions [36]. As in our earlier studies [20, 21], $B$ is chosen such that the phase transition temperature is $T_{c}=165 \mathrm{MeV}$. We assume full kinetic and chemical equilibrium for both phases throughout the temperature range considered in this work.

Final hadron spectra are calculated through the Cooper-Frye decoupling procedure [5] as particle emis- sion from a constant- $T$ surface obtained from the hydrodynamic calculation. We determine the decoupling temperature $T_{\mathrm{dec}}-$ a parameter which controls the $p_{T}$ slopes of the hadron spectra in the single- $T_{\mathrm{dec}}$ hydrodynamic framework - from the RHIC data, as described in Refs. [20, 21]. For a recent discussion of the relation of $T_{\mathrm{dec}}$ and decoupling dynamics, see [37, 38]. After the Cooper-Frye decoupling, all 2- and 3-body strong and electromagnetic decays of unstable hadronic states are accounted for. Thus, the feed-down from weak decays is not included in this work.

\section{INITIAL STATE AND CENTRALITY SELECTION}

As initial conditions, the boost-invariant hydrodynamic calculation, at an impact parameter $b$, requires the densities $\epsilon\left(x, y, \tau_{0} ; b\right), n_{B}\left(x, y, \tau_{0} ; b\right)$ at an initial time $\tau_{0}$. Our reference baseline is central collisions, for which we obtain the initial densities from the EKRT minijet (final state) saturation model 22]. In addition to the primary partonic transverse energy and the net-baryon number produced at midrapidity in central AA collisions, the EKRT model also gives the average formation time in terms of the saturation momentum, $\tau_{f}=1 / p_{\text {sat }}$.

Assuming immediate thermalization at production, $\tau_{0}=\tau_{f}$, using the binary collision (BC) profiles for the transverse-coordinate dependence of the densities, and setting $T_{\mathrm{dec}}=150 \mathrm{MeV}$, we have previously shown that the minijet + saturation + hydrodynamics model is in good agreement with the RHIC data in most central $\mathrm{Au}+\mathrm{Au}$ collisions [20, 21]. We have also demonstrated that using the wounded nucleon (WN) profiles and $T_{\mathrm{dec}}=140 \mathrm{MeV}$ leads to practically equally good results 37]. Also predictions for the central $\mathrm{Pb}+\mathrm{Pb}$ collisions at the LHC have been presented [20].

The determination of the transverse profiles for $\epsilon$ and $n_{B}$ is, however, problematic for the following reasons: First, the simplest version of the EKRT model for headon collisions has only one saturation momentum scale, $p_{\text {sat }}$, which is perturbative, $\sim 1-2 \mathrm{GeV}$ for large nuclei at RHIC and LHC. In the localized version [39] $p_{\text {sat }}$ depends on the transverse location but near the edges of the system, where the produced matter density becomes low enough, $p_{\text {sat }}$ becomes non-perturbative and the minijet calculation unreliable. Second, non-central collisions can be expected to constitute a multiscale problem as very different nuclear regions are colliding with each other, and we cannot expect the simple EKRT model to describe the more peripheral collisions very well. Third, as discussed in [39, 40], different regions in the transverse plane obviously form at different times; the center with a larger saturation scale forms earlier than the edges of the system. This phenomenon adds the complication of needing to provide the initial conditions on a surface $\tau=\tau_{0}(x, y)$ instead of just at a fixed initial time $\tau=\tau_{0}$ (see the discussion in [41]). 
It is then obvious that some additional modeling is necessary in fixing the initial transverse profiles of $\epsilon$ and $n_{B}$ for non-central collisions in particular. One possibility is to assume a mixture of the $\mathrm{BC}$ and $\mathrm{WN}$ profiles, either for the energy density or the entropy density [41], with the mixture coefficient determined from RHIC data. Then, however, since the initial transverse profiles should be affected by production dynamics as discussed in [40], an uncertainty in the extrapolation to the LHC energies would remain anyway since the mixture coefficients can change from RHIC to the LHC.

Instead of introducing further model details and thus also further model uncertainties regarding the transverse profiles and their $\sqrt{s}$ dependence, we choose a simpler and more transparent approach: We fix the initial time $\tau_{0}$ to the value computed from the EKRT model in central collisions, and we compute the spectra and elliptic flow coefficients with the BC and WN initializations separately, considering these as two limiting cases of transverse profiles both at RHIC and at the LHC.

Our BC initial conditions for $A+A$ collisions are analogous to the eBC model of Ref. [41] and are given by

$$
\begin{aligned}
\epsilon_{\mathrm{BC}}(\mathbf{r} ; \mathbf{b}) & =\frac{1}{\tau_{0}} \frac{[d E / d \eta]_{b=0}}{N_{\mathrm{BC}}^{A A}(0)} \frac{d N_{\mathrm{BC}}^{A A}}{d^{2} \mathbf{r}}(\mathbf{r} ; \mathbf{b}) \\
n_{B}^{\mathrm{BC}}(\mathbf{r} ; \mathbf{b}) & =\frac{1}{\tau_{0}} \frac{\left[d N_{B} / d \eta\right]_{b=0}}{N_{\mathrm{BC}}^{A A}(0)} \frac{d N_{\mathrm{BC}}^{A A}(\mathbf{b})}{d^{2} \mathbf{r}}(\mathbf{r} ; \mathbf{b}),
\end{aligned}
$$

where $[d E / d \eta]_{b=0}$ and $\left[d N_{B} / d \eta\right]_{b=0}$ and $\tau_{0}$ are obtained from the EKRT model [20] for central collisions and where the $\mathrm{BC}$ profile is

$$
\frac{d N_{\mathrm{BC}}^{A A}}{d^{2} \mathbf{r}}(\mathbf{r} ; \mathbf{b})=T_{A}\left(\mathbf{r}+\frac{\mathbf{b}}{2}\right) T_{A}\left(\mathbf{r}-\frac{\mathbf{b}}{2}\right) \sigma_{N N}^{\mathrm{in}},
$$

and $N_{\mathrm{BC}}^{A A}(0)=\sigma_{N N}^{\mathrm{in}} \int d^{2} \mathbf{r}\left[T_{A}(\mathbf{r})\right]^{2}$. In computing the standard nuclear thickness functions $T_{A}$, we use the Woods-Saxon parametrization of the nuclear density. Similarly, our WN initial conditions are analogous to the eWN model of Ref. [41] and computed from

$$
\begin{aligned}
\epsilon_{\mathrm{WN}}(\mathbf{r} ; \mathbf{b}) & =C_{\epsilon} \frac{d N_{\mathrm{WN}}^{A A}}{d^{2} \mathbf{r}}(\mathbf{r} ; \mathbf{b}) \\
n_{B}^{\mathrm{WN}}(\mathbf{r} ; \mathbf{b}) & =C_{B} \frac{d N_{\mathrm{WN}}^{A A}}{d^{2} \mathbf{r}}(\mathbf{r} ; \mathbf{b}),
\end{aligned}
$$

where the normalization constants $C_{\epsilon}$ and $C_{B}$ are fixed by requiring, for central collisions, the initial entropy $d S / d \eta$ and the initial net-baryon number in the eWN initial state to be the same as in the BC initial state. The WN profile is given by

$$
\begin{aligned}
& \frac{d N_{\mathrm{WN}}}{d^{2} \mathbf{r}}(\mathbf{r} ; \mathbf{b}) \\
= & T_{A}\left(\mathbf{r}+\frac{\mathbf{b}}{2}\right)\left[1-\left(1-\frac{\sigma_{N N}^{\mathrm{in}}}{A} T_{A}\left(\mathbf{r}-\frac{\mathbf{b}}{2}\right)\right)^{A}\right] \\
+ & T_{A}\left(\mathbf{r}-\frac{\mathbf{b}}{2}\right)\left[1-\left(1-\frac{\sigma_{N N}^{\mathrm{in}}}{A} T_{A}\left(\mathbf{r}+\frac{\mathbf{b}}{2}\right)\right)^{A}\right] .
\end{aligned}
$$

The initial state parameters for the different collision energies are shown in Table I. The integral of $d N_{W N} / d^{2} \mathbf{r}$ over the transverse plane is the number of participants, $N_{\text {part }}(\mathbf{b})$, for a collisions at impact parameter $\mathbf{b}$.

\begin{tabular}{lcc}
\hline \hline & RHIC & LHC \\
$\sqrt{s_{N N}}[\mathrm{GeV}]$ & 200 & 5500 \\
$\tau_{0}[\mathrm{fm}]$ & 0.17 & 0.097 \\
{$[d E / d \eta]_{b=0}[\mathrm{GeV}]$} & 2460 & 14800 \\
{$\left[d N_{B} / d \eta\right]_{b=0}$} & 15.3 & 3.36 \\
$\sigma_{N N}^{i n}[\mathrm{mb}]$ & 42 & 60 \\
\hline \hline
\end{tabular}

TABLE I: The initial state parameters for $\mathrm{Au}+\mathrm{Au}$ collisions at RHIC and $\mathrm{Pb}+\mathrm{Pb}$ at the LHC.

In other words, we assume here that once the EKRT model fixes the normalizations of $\epsilon$ and $n_{B}$ for central collisions, the optical Glauber model gives the impact parameter dependence of the initial conditions, assuming proportionality either to the $\mathrm{BC}$ or $\mathrm{WN}$ densities. We would like to emphasize that in this work we consider the two limits as they are. We do not try to fit the RHIC data by finding the best linear combination of the $\mathrm{eBC}$ and eWN profiles. Instead, we show that the RHIC data fall between the two limits and take the difference to represent the uncertainty in the extrapolation to the LHC energy.

Qualitatively, there are two main differences between the two initializations. First, the number of BCs drops much faster with increasing impact parameter than the number of WNs. This leads to a faster dropping multiplicity for the eBC than for the eWN initialization as a function of centrality. Second, at a given impact parameter the $\mathrm{BC}$ density in the transverse plane falls faster as a function of the transverse distance from the center of the fireball than the WN density. At a given multiplicity, this leads to stronger transverse pressure gradients for the eBC than for the eWN initialization. Thus, if the same decoupling condition (same $T_{\mathrm{dec}}$ ) were used, the $\mathrm{eBC}$ initialization would lead to both stronger transverse flow and larger elliptic flow than the eWN initialization.

To discuss the centrality classes, we apply the optical Glauber model, where the total cross section is given by

$$
\sigma_{\text {tot }}^{A A}=\int d^{2} \mathbf{b} \frac{d \sigma_{\mathrm{tot}}}{d^{2} \mathbf{b}}=\int d^{2} \mathbf{b}\left[1-e^{T_{A A}(\mathbf{b}) \sigma_{N N}^{\mathrm{in}}}\right],
$$

where $T_{A A}$ is the standard nuclear overlap function. For the centrality classes considered here, $c_{1}=0-5 \%$, $c_{2}=5-10 \%, c_{3}=10-15 \%, \ldots$, we find the impact parameter ranges $\left[0, b_{1}\right],\left[b_{1}, b_{2}\right], \ldots$ such that, e.g., for the 2 nd centrality class $c_{2}$

$$
\sigma_{c_{2}}^{A A} / \sigma_{\mathrm{tot}}^{A A}=0.05=\int_{b_{1}}^{b_{2}} d^{2} \mathbf{b}\left[1-e^{T_{A A}(\mathbf{b}) \sigma_{N N}^{\mathrm{in}}}\right]
$$

and similarly for the other classes $c_{i}$. Using the $d \sigma_{\text {tot }} / d^{2} b$ as the weight, we determine the average impact parame- 
ter for each centrality class as follows

$$
\langle b\rangle_{i}=\frac{1}{\sigma_{\mathrm{C}_{\mathrm{i}}}^{A A}} \int_{b_{i-1}}^{b_{i}} d^{2} \mathbf{b}\left[1-e^{T_{A A}(\mathbf{b}) \sigma_{N N}^{\mathrm{in}}}\right] b .
$$

The average number of participants in a given centrality class is computed similarly. The initial densities for each centrality class are then computed using $b=\langle b\rangle_{i}$ in Eqs. 1 and 3. The average impact parameters and average numbers of participants for selected centrality classes are shown in Table II.

\begin{tabular}{cccccc}
\hline \hline & \multicolumn{2}{c}{ RHIC } & \multicolumn{2}{c}{ LHC } \\
\cline { 2 - 3 } \cline { 5 - 6 } centrality $\%$ & $b[\mathrm{fm}]$ & $N_{\text {part }}$ & & $b[\mathrm{fm}]$ & $N_{\text {part }}$ \\
\hline $0-5$ & 2.24 & 347 & 2.31 & 374 \\
$5-10$ & 4.09 & 289 & 4.23 & 315 \\
$10-15$ & 5.30 & 242 & 5.47 & 264 \\
$15-20$ & 6.27 & 202 & 6.48 & 221 \\
$20-30$ & 7.49 & 153 & 7.74 & 168 \\
$30-40$ & 8.87 & 102 & 9.17 & 112 \\
$40-60$ & 10.6 & 50.8 & 10.9 & 56.7 \\
$60-70$ & 12.1 & 19.6 & 12.5 & 21.2 \\
$70-80$ & 13.0 & 9.13 & 13.4 & 9.65 \\
\hline \hline
\end{tabular}

TABLE II: The average impact parameters and the average numbers of participants in selected centrality classes from the optical Glauber model.

\section{ELLIPTIC FLOW, ECCENTRICITIES AND TRANSVERSE FLOW}

The transverse momentum and rapidity dependent Fourier coefficients $v_{n}$ for each centrality class are defined as

$$
v_{n}\left(y, p_{T} ; b\right) \equiv\left(\frac{d N(b)}{d y d p_{T}^{2}}\right)^{-1} \int_{-\pi}^{\pi} d \phi \cos (n \phi) \frac{d N(b)}{d y d p_{T}^{2} d \phi}
$$

while the $p_{T}$-integrated $v_{n}$ 's are given by

$$
v_{n}(y ; b) \equiv\left(\frac{d N(b)}{d y}\right)^{-1} \int_{-\pi}^{\pi} d \phi \cos (n \phi) \frac{d N(b)}{d y d \phi},
$$

where $b$ stands for the average impact parameter $\langle b\rangle_{i}$ in the centrality class $c_{i}$. Due to the longitudinal boost symmetry assumed here, the $v_{n}$ coefficients, which we compute from the hydrodynamic spectra, do not depend on rapidity, thus $y=0$ is implicit. Our definition of the minimum bias elliptic flow coefficient, $v_{2}^{\text {m.bias }}\left(y, p_{T}\right)$, in turn is as follows,

$$
v_{2}^{\mathrm{m} . \operatorname{bias}}\left(y, p_{T}\right) \equiv \frac{\int d^{2} \mathrm{~b} v_{2}\left(y, p_{T} ; b\right) \frac{d N(b)}{d y d p_{T}^{2}}}{\int d^{2} \mathrm{~b} \frac{d N(b)}{d y d p_{T}^{2}}} .
$$

In the calculations the impact parameter is in the $x$ direction. Therefore, the system is initially shorter and the pressure gradients stronger in the $x$ direction. Stronger pressure gradients will generate stronger transverse flow during the evolution in the short direction. As a result the system grows faster in the $x$ direction than in the $y$ direction. The generated flow asymmetry manifests in the azimuthal dependence of hadron spectra and a nonzero $v_{2}$ is observed.

Figures 1 and 2 show the QGP-MP and MP-HRG phase boundaries and the decoupling boundaries in the $x$ and $y$ directions from our hydrodynamical simulations with $b=7.49 \mathrm{fm}$ for RHIC and $b=7.74 \mathrm{fm}$ for the LHC, both corresponding to the $20-30 \%$ centrality class. Both the RHIC and LHC results are shown with two different initializations, the eBC and eWN, discussed in the previous section. Lifetimes of the different phases at different transverse locations can be read off from Figs. 1 and 2 in each case. With the same collision energy different initial profiles correspond to slightly different multiplicity. Hence there is a difference e.g. in the initial transverse size of the eBC and eWN initializations in the figures. The first-order phase transition, present in the EoS we use, produces a shock wave at the boundary of HRG and MP, which in turn causes the peaks at the edge of the system seen especially in Fig. 2. However, contribution to the hadronic observables from the peaks is small. We have checked that removing the peaks causes less than $5 \%$ changes in the hadron spectra and elliptic flow coefficients.

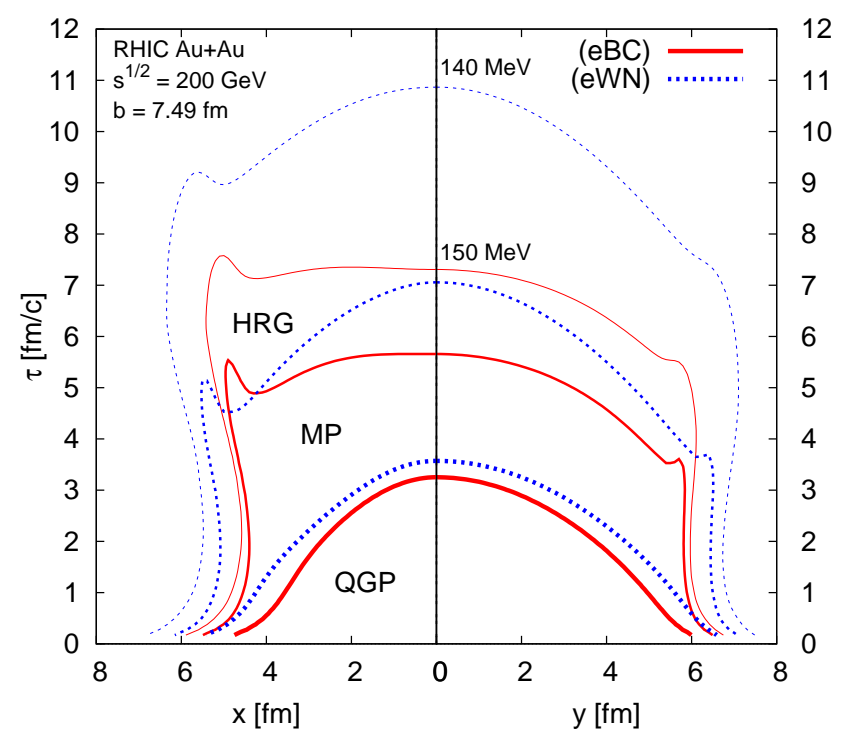

FIG. 1: (Color online) The phase boundaries and the constant- $T_{\mathrm{dec}}$ decoupling curves from our calculation for $\sqrt{s_{N N}}=200 \mathrm{GeV} \mathrm{Au}+\mathrm{Au}$ collisions at RHIC in the 20$30 \%$ centrality class. Both $x$ and $y$ directions are shown [16].

A convenient way to illustrate the global features of the hydrodynamical space-time evolution of the matter is through the following three quantities: spatial eccentricity, momentum-space eccentricity and average transverse flow. These quantities are introduced in Ref. [7], 


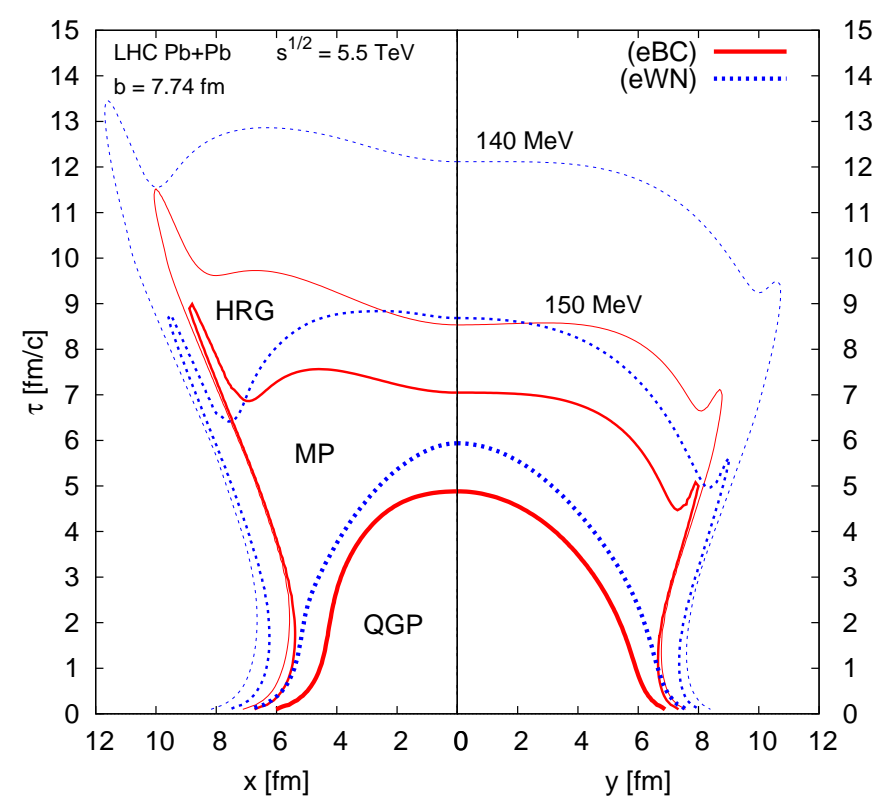

FIG. 2: (Color online) The Phase boundaries and the constant- $T_{\text {dec }} \mathrm{MeV}$ decoupling curves for $\sqrt{s_{N N}}=5500 \mathrm{GeV}$ $\mathrm{Pb}+\mathrm{Pb}$ collisions at the LHC in the $20-30 \%$ centrality class.

where also their behavior for different systems has been discussed. The spatial eccentricity is defined as

$$
\varepsilon_{x} \equiv \frac{\left\langle y^{2}-x^{2}\right\rangle}{\left\langle y^{2}+x^{2}\right\rangle} \equiv \frac{\int d x d y \epsilon(x, y, \tau)\left(y^{2}-x^{2}\right)}{\int d x d y \epsilon(x, y, \tau)\left(y^{2}+x^{2}\right)},
$$

where the integral is over the transverse plane and the energy density $\epsilon$ is used as the weighting factor. Similarly, the momentum space eccentricity is defined as

$$
\varepsilon_{p} \equiv \frac{\int d x d y\left(T^{x x}-T^{y y}\right)}{\int d x d y\left(T^{x x}+T^{y y}\right)}
$$

where $T^{i i}$ are the components of the energy-momentum tensor. The average transverse flow can be defined as

$$
\left\langle v_{T}\right\rangle \equiv \frac{\left\langle\gamma v_{T}\right\rangle}{\langle\gamma\rangle} \equiv \frac{\int d x d y \epsilon(x, y, \tau) \gamma v_{T}(x, y, t)}{\int d x d y \epsilon(x, y, \tau) \gamma}
$$

where $v_{T}=\sqrt{v_{x}^{2}+v_{y}^{2}}$ and the $\gamma$ factor has been used as an additional weight.

The spatial eccentricity $\varepsilon_{x}$ measures the asymmetry of the matter distribution in the $x$ and $y$ directions, thus it also measures the asymmetry of pressure gradients. In a noncentral heavy ion collision, with the impact parameter along the $x$-axis, the initial distribution of the matter corresponds to a positive initial $\varepsilon_{x}$ which acts as a driving force to an asymmetric transverse flow. The generated flow asymmetry is quantified by $\varepsilon_{p}$. The sign convention of $\varepsilon_{x}$ and $\varepsilon_{p}$ is such that an initially positive $\varepsilon_{x}$ leads to a positive $\varepsilon_{p}$. A positive $\varepsilon_{p}$ at the end of the evolution will convert to a positive elliptic flow coefficient $v_{2}$, which can be extracted from hadron spectra. The value of $\varepsilon_{p}$, when the system decouples, is approximately twice the $p_{T}$-integrated $v_{2}$ for pions [7], see Figs. 3 and 5. During the evolution the established asymmetric flow field tends to drive $\varepsilon_{x}$ towards zero, i.e. towards azimuthally symmetric matter distribution. Therefore the driving force for the growth of elliptic flow is strongest during the early stages of the collision. At later times $\varepsilon_{x}$ goes through zero and eventually to slightly negative values and $\varepsilon_{p}$ will not increase anymore.

Figure 3 shows the time evolution of $\varepsilon_{x}, \varepsilon_{p}$ and $\left\langle v_{T}\right\rangle$ from the same calculation as the curves in Figs. 1 and 2. Initially $\varepsilon_{x}$ is very similar for RHIC and the LHC if the same initialization profiles (eWN or eBC) are used. At the beginning of the evolution, when most of the matter is in the QGP phase, the flow generated by the pressure gradients starts to decrease $\varepsilon_{x}$. Simultaneously $\varepsilon_{p}$ is increasing. The initial rates of change for $\varepsilon_{x}$ and $\varepsilon_{p}$ are very similar at RHIC and the LHC. During the QGP phase there is no significant difference in the behavior of $\varepsilon_{x}$ and $\varepsilon_{p}$ between the two collision energies, but much bigger difference between the two chosen initial profiles. When the system enters the mixed phase all pressure gradients vanish ceasing the increase of $\varepsilon_{p}$ and $\left\langle v_{T}\right\rangle$, i.e. $\varepsilon_{p}$ saturates even before $\varepsilon_{x}$ goes to zero. During the mixed phase the matter is not accelerating, but the established flow field still expands the system transversally. This will cause $\varepsilon_{p}$ actually to decrease during the mixed phase. The transition times between the different phases for different initializations can be read off from Figs. 1 and 2. These times coincide with the structures seen in the behavior of $\varepsilon_{p}$, and $\left\langle v_{T}\right\rangle$. Transitions between the phases happen at different times at different transverse locations, therefore e.g. $\left\langle v_{T}\right\rangle$ never completely saturates when most of the matter is in the mixed phase: part of the matter is always either in the HRG or in QGP phase, where the pressure gradients do not vanish.

A significant difference between the LHC and RHIC is the lifetime of the QGP phase. At the LHC $\varepsilon_{p}$ has more time to grow before the system enters the mixed phase, which leads to a larger $\varepsilon_{p}$ at the end of the evolution. Therefore we also expect that the elliptic flow coefficient $v_{2}$ will be larger at the LHC than at RHIC. Longer lifetime of the QGP phase reflects also in the behavior of $\varepsilon_{x}$ and $\left\langle v_{T}\right\rangle$. At RHIC $\varepsilon_{p}$ saturates well before $\varepsilon_{x}$ goes to zero, and $\varepsilon_{x}$ is still positive when the system enters the HRG phase and $\varepsilon_{p}$ is slightly increasing. Therefore there could still be some elliptic flow generated during the HRG phase at RHIC, i.e. $v_{2}$ would have some sensitivity on the decoupling condition. This can be verified by an explicit calculation and within our framework $v_{2}$ increases by $\sim 20-30 \%$ at $N_{\text {part }} \sim 150$ when the decoupling temperature is changed from $T_{\mathrm{dec}}=160 \mathrm{MeV}$ to $T_{\text {dec }}=130 \mathrm{MeV}$. On the other hand at the LHC the saturation of $\varepsilon_{p}$ and the sign change of $\varepsilon_{x}$ happen more or less simultaneously and when the system enters the HRG phase $\varepsilon_{x}$ is already negative and $\varepsilon_{p}$ is slightly decreasing. At the LHC $v_{2}$ is slightly decreasing when $T_{\text {dec }}$ is de- 

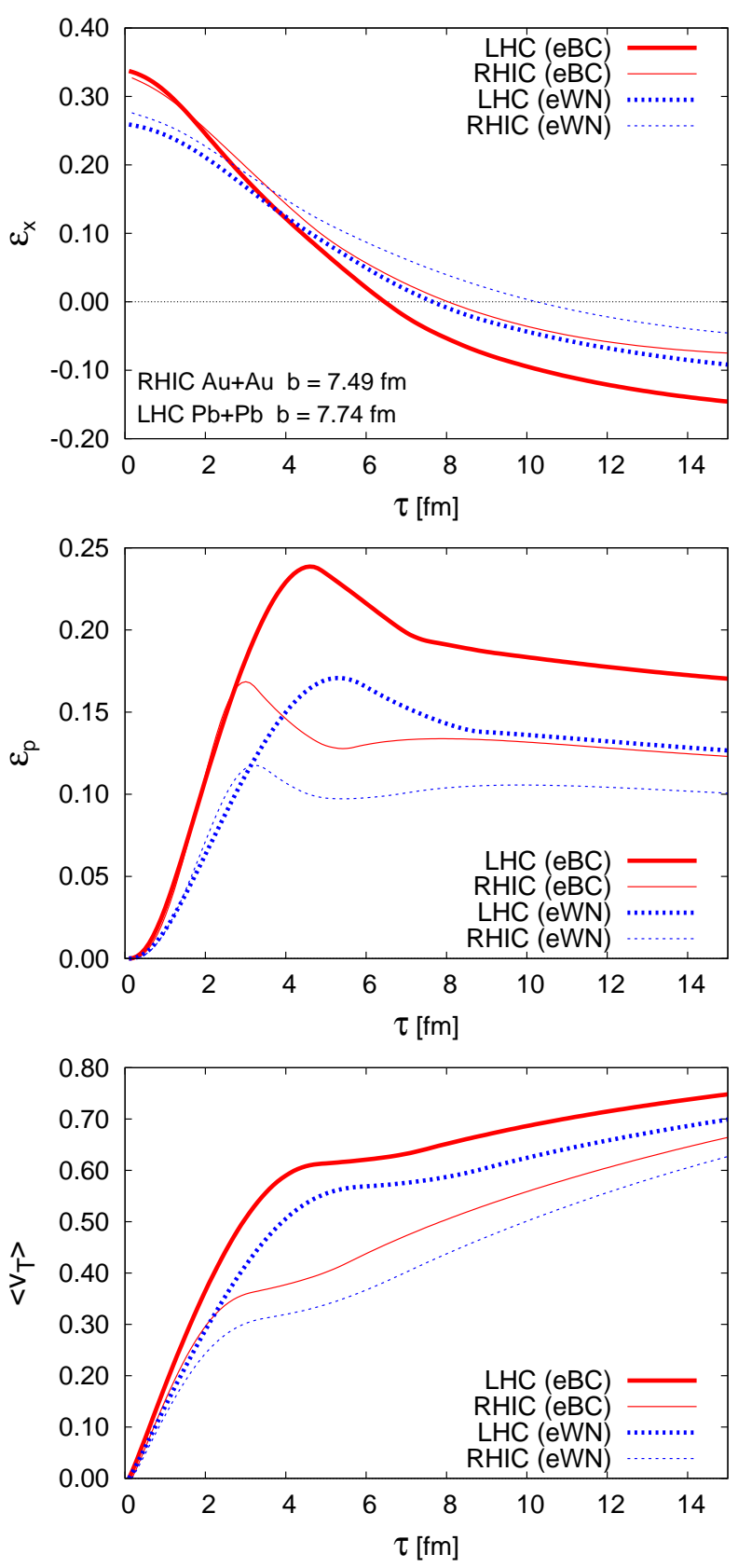

FIG. 3: (Color online) The spatial and momentum space eccentricity and the average transverse velocity as function of the longitudinal proper time for $\sqrt{s_{N N}}=200 \mathrm{GeV}$ $\mathrm{Au}+\mathrm{Au}$ collisions at RHIC (thin solid and dashed curves) and $\sqrt{s_{N N}}=5500 \mathrm{GeV} \mathrm{Pb}+\mathrm{Pb}$ collisions at the LHC (thick solid and dashed curves) in the $20-30 \%$ centrality class.

creasing but the HRG effects are significantly smaller at the LHC than at RHIC. Thus the predictions for the $p_{T^{-}}$ integrated $v_{2}$ for the LHC are insensitive to the details of the HRG dynamics and therefore quite robust once the initial profile is fixed.

In contrast to the behavior of $\varepsilon_{p},\left\langle v_{T}\right\rangle$ never completely saturates. Even at late stages of the collision $\left\langle v_{T}\right\rangle$ can still increase significantly. Although the azimuthal asymmetry of the pressure gradients almost vanishes, the pressure gradient in the radial direction does not vanish. Even though the gradient is small in the HRG phase, additional transverse velocity is generated and can be easily seen in the slopes of hadron $p_{T}$ spectra. Thus the $p_{T}$ spectra of hadrons remain sensitive to the decoupling temperature and HRG dynamics both at RHIC and the LHC. During the QGP phase the pressure gradients are strongest and the longer lifetime of the QGP phase at the LHC leads to a clearly larger transverse flow before the mixed phase than at RHIC. Regardless of the decoupling condition the $p_{T}$ spectra at the LHC are always flatter than at RHIC.

The behavior of the $p_{T}$-integrated $v_{2}$ for different initializations can be quite easily determined from the behavior of $\varepsilon_{p}$ alone. The differential elliptic flow coefficient $v_{2}\left(p_{T}\right)$ however depends not only on $\varepsilon_{p}$, but also on the slopes of the hadron $p_{T}$ spectra, which in turn depend on $\left\langle v_{T}\right\rangle$ and temperature. In general, increasing $\varepsilon_{p}$ will increase $v_{2}\left(p_{T}\right)$ at fixed $p_{T}$ but increasing $\left\langle v_{T}\right\rangle$ will decrease it [42]. The net effect depends on the details of the HRG dynamics. Within our framework with full chemical and kinetic equilibrium in the HRG throughout the evolution, the net result is that $v_{2}\left(p_{T}\right)$ is quite insensitive to the decoupling temperature. At RHIC $v_{2}\left(p_{T}\right)$ is slightly increasing and at the LHC slightly decreasing with decreasing $T_{\text {dec }}$. These changes are, however, small and practically within our framework, predictions for $v_{2}\left(p_{T}\right)$ are independent of the decoupling temperature. Changes in the HRG dynamics would change this behavior, e.g. chemical freeze-out before kinetic freeze-out [8, 9, 10] would modify the dependence of spectral slopes on temperature and flow conditions and thus would affect the behavior of $v_{2}\left(p_{T}\right)$ as a function of the decoupling condition. These effects are, however, not studied in this work.

\section{RESULTS FOR RHIC}

Figure 4 shows the calculated pion spectra for different centrality classes in $\sqrt{s_{N N}}=200 \mathrm{GeV} \mathrm{Au}+\mathrm{Au}$ collisions at RHIC, compared with the PHENIX data [43]. Shown in the figure are the results corresponding to the two different initializations we consider here. The decoupling temperature is fixed to $T_{\mathrm{dec}}=150 \mathrm{MeV}$ for the eBC initialization, as in our previous works [20, 21]. As seen in Fig. 3, the eWN initialization generates less transverse flow for a given decoupling condition, therefore - in order to reproduce the pion $p_{T}$ spectra - the system must be allowed to decouple later than for the eBC initialization. In the eWN case, $T_{\text {dec }}=140 \mathrm{MeV}$ describes the data well. With this difference in $T_{\mathrm{dec}}$, both initializations give an equally good agreement with the data for central and mid-central collisions. For more peripheral collisions the calculations start to separate and, as expected, the eBC results fall below the eWN results but 


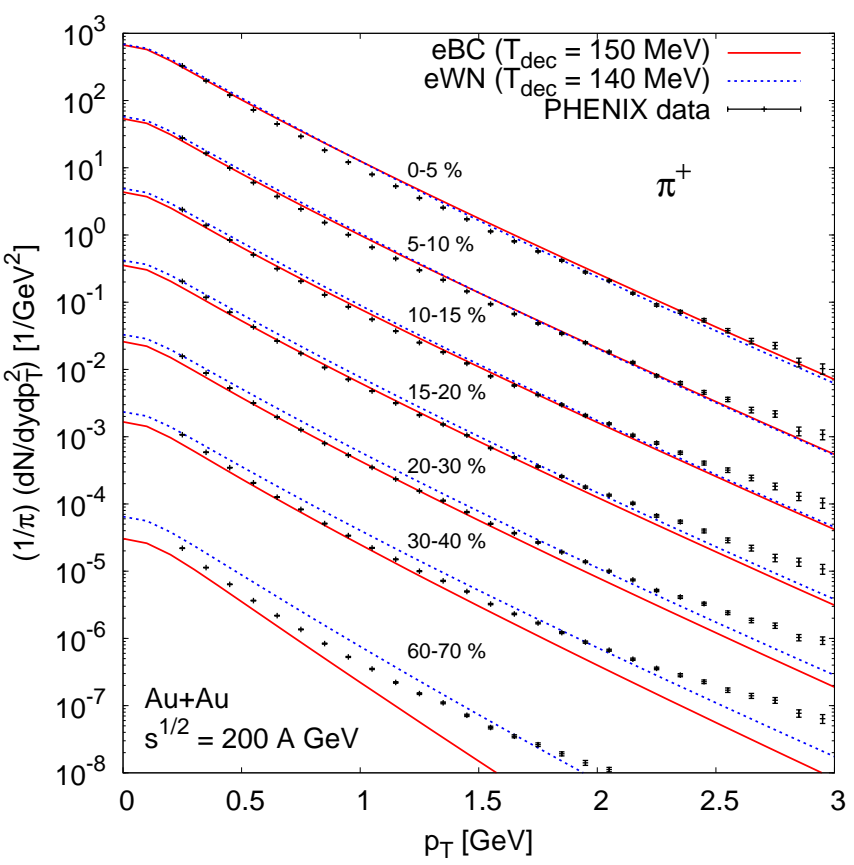

FIG. 4: (Color online) The $p_{T}$ spectra of positive pions for $\sqrt{s_{N N}}=200 \mathrm{GeV} \mathrm{Au}+\mathrm{Au}$ collisions at RHIC compared with the PHENIX data [43]. The solid (dashed) lines are for the $\mathrm{eBC}(\mathrm{eWN})$ initialization. The centrality classes are indicated in the figure and are scaled by the increasing powers of $10^{-1}$.

the data lies still well between the two limits considered. Fig. 4 also demonstrates how in most central collisions the hydrodynamically computed pion spectra reproduce the data well in the region $p_{T} \lesssim 3 \mathrm{GeV}$, while the applicability region of hydrodynamic spectra in the 60-70\% centrality class is limited to $p_{T} \lesssim 1.5 \mathrm{GeV}$, only.

Figure 5 shows our model calculations of the $p_{T^{-}}$ integrated $v_{2}$ for charged hadrons as a function of the number of participants, compared with the PHOBOS data [44]. For mid-peripheral collisions $\left(N_{\text {part }} \sim 150-\right.$ 200) the agreement with the data is quite good. However, for the most central classes we underestimate the data - a typical feature also in several other hydrodynamical models. The underestimation of $v_{2}$ is usually associated with fluctuations in the initial geometry of the system [45], which are not accounted for in our treatment. For very peripheral collisions the model eventually starts to overshoot the data but in these collisions, with the small system sizes and particle numbers, we expect, in any case, the validity of hydrodynamic modeling to deteriorate. From mid-peripheral to central collisions the $\mathrm{eBC}$ initialization generates more elliptic flow than the eWN initialization, as expected on the basis of Fig. 3. This results from the larger pressure gradient in the eBC initialization. However, for more peripheral collisions the behavior changes and the eBC results fall below the eWN ones. Since the total multiplicity for the eBC initial state drops faster with increasing impact parameter, it also causes the lifetime of the QGP phase to

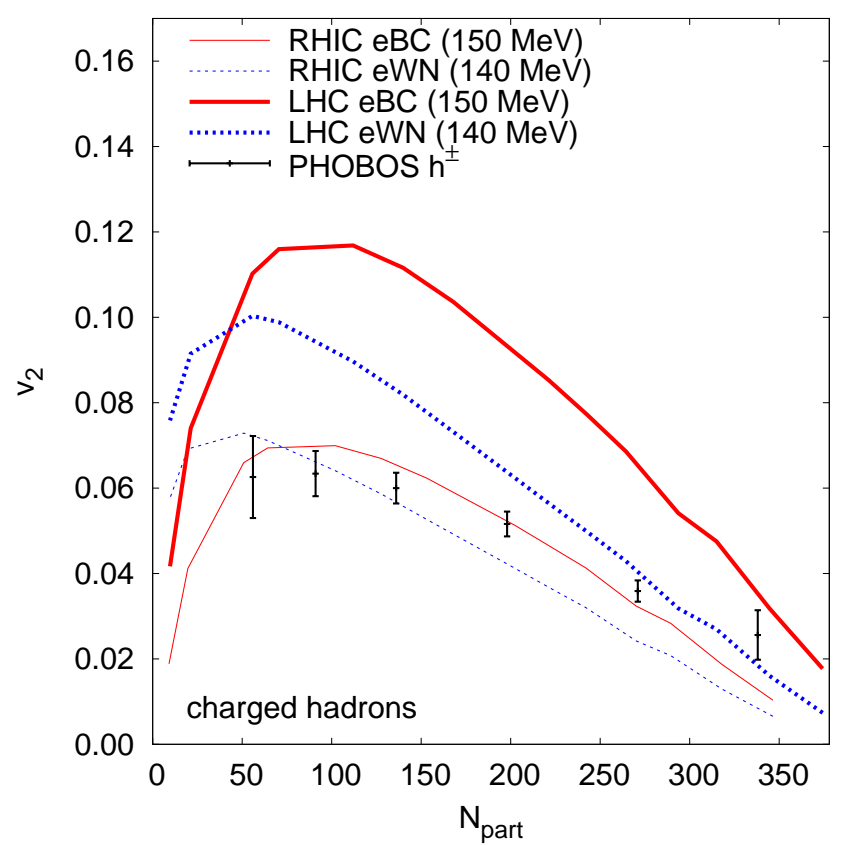

FIG. 5: (Color online) The $p_{T}$-integrated $v_{2}$ of charged hadrons for $\sqrt{s_{N N}}=200 \mathrm{GeV} \mathrm{Au}+\mathrm{Au}$ collisions at RHIC (thin lines) and $\sqrt{s_{N N}}=5500 \mathrm{GeV} \mathrm{Pb}+\mathrm{Pb}$ collisions at the LHC (thick lines) vs. the number of participants. The data is from the PHOBOS collaboration [44].

drop faster. For very peripheral collisions the lifetime of the $\mathrm{eBC}$ system is considerably shorter than that of the eWN system. Thus the elliptic asymmetry will not have time to develop before freeze-out. Also the pressure gradients in the $\mathrm{eBC}$ case are larger than in the eWN case for central and mid-peripheral collisions, but become very similar for peripheral collisions. As a result, the elliptic flow predicted by the eBC initialization will eventually drop below the eWN results as we go towards more peripheral collisions. At RHIC the results coincide when $N_{\text {part }} \sim 70$. In addition to the RHIC results, Fig. 5 shows also the LHC prediction, which we shall comment on shortly.

Figure 6 shows our results for the minimum bias $v_{2}\left(p_{T}\right)$ of pions in $\mathrm{Au}+\mathrm{Au}$ collisions at RHIC (thin lines), compared with the RHIC data from STAR [46] and PHENIX [47]. The agreement with the data is quite good up to $p_{T} \lesssim 1.5 \mathrm{GeV}$ for both initial profiles. As is the case with the $p_{T}$-integrated $v_{2}$, also $v_{2}\left(p_{T}\right)$ is larger for the $\mathrm{eBC}$ than the eWN initialization from mid-peripheral to central collisions. However, for peripheral collisions also $v_{2}\left(p_{T}\right)$ with the eBC initialization eventually falls below the eWN results for the reasons discussed above. This can be seen in Fig. 5 as the crossing of the eWN and eBC curves for RHIC. For the minimum bias $v_{2}\left(p_{T}\right)$ all centrality classes between $0-80 \%$ are included and the net result is that $v_{2}\left(p_{T}\right)$ averages nearly to the same values with both initializations at RHIC. 


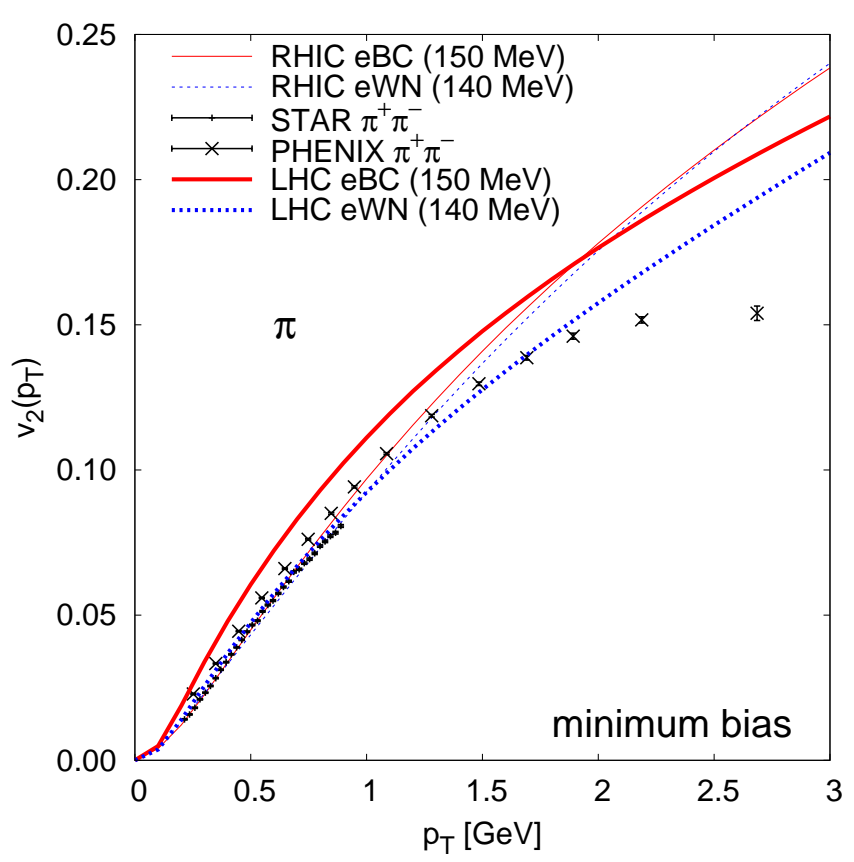

FIG. 6: (Color online) The minimum bias $v_{2}\left(p_{T}\right)$ of pions for $\sqrt{s_{N N}}=200 \mathrm{GeV} \mathrm{Au}+\mathrm{Au}$ collisions at RHIC (thin lines) and $\sqrt{s_{N N}}=5500 \mathrm{GeV} \mathrm{Pb}+\mathrm{Pb}$ collisions at the LHC (thick lines) as a function of transverse momentum. The data is from the PHENIX [47] and STAR [46] collaborations.

\section{PREDICTIONS FOR THE LHC}

In Fig. 7 we show our prediction for the transverse momentum spectra of positive pions for different centrality classes in $\sqrt{s_{N N}}=5.5 \mathrm{TeV} \mathrm{Pb}+\mathrm{Pb}$ collisions at the LHC. The charged hadron multiplicity in the most central collisions is ca. 2600 [20]. Once the framework is tested in RHIC $\mathrm{Au}+\mathrm{Au}$ collisions, the only freedom left is the choice of the initial profile and of the decoupling temperature $T_{\text {dec }}$. For the initial profile we consider the same limits as at RHIC, i.e. we do calculations with both the eWN and eBC profiles, with normalization fixed from the EKRT model for central collisions. As discussed in the context of dynamical decoupling [37], we do not expect $T_{\text {dec }}$ to change significantly from RHIC to the LHC. Thus we show the eBC results with $T_{\text {dec }}=150 \mathrm{MeV}$ and the eWN results with $T_{\text {dec }}=140 \mathrm{MeV}$. Both initializations give similar results for central and mid-peripheral collisions but, similarly to the RHIC case, the two calculations start to separate for more peripheral collisions.

In addition to the RHIC results, we show our predictions for the LHC in Fig. 5 for the $p_{T}$-integrated $v_{2}$ and in Fig. 6 for the minimum bias $v_{2}\left(p_{T}\right)$, both for the eBC and eWN initializations. From Fig. 5 we see that, as expected, the $p_{T}$-integrated $v_{2}$ is much larger at the LHC than at RHIC. Due to the longer lifetime of the QGP at the LHC the flow asymmetry has more time to develop before the system goes into the mixed phase where pressure gradients vanish.

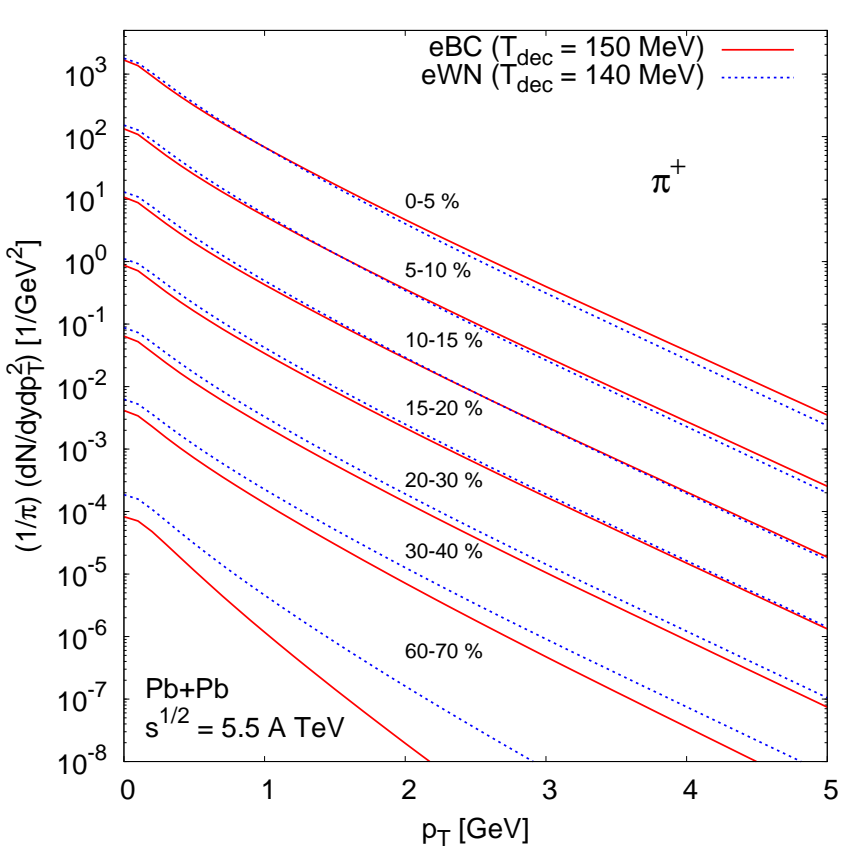

FIG. 7: (Color online) Our predictions for the transverse momentum spectra of positive pions for $\sqrt{s_{N N}}=5500 \mathrm{GeV} \mathrm{Pb}$ $+\mathrm{Pb}$ collisions at the LHC. The results with the eBC (eWN) initialization are shown by solid (dashed) lines. The centrality classes are the same as for RHIC in Fig. 4.

Although the $p_{T}$-integrated $v_{2}$ is clearly larger at the LHC than at RHIC, the situation is not as clear for the minimum bias $v_{2}\left(p_{T}\right)$. For the eBC initialization our LHC prediction is clearly above all the RHIC data points and, in the small- $p_{T}$ region, also above the RHIC predictions. On the other hand, in the low- $p_{T}$ region the eWN results for the LHC are very close to the $v_{2}\left(p_{T}\right)$ values at RHIC. This does not contradict the results for the $p_{T^{-}}$ integrated $v_{2}$, which are obtained by weighting with the particle's $p_{T}$ spectra. Since the spectra at the LHC are much flatter than at RHIC (,i.e. the average $p_{T}$ is larger at the LHC), $v_{2}$ gets more weight from the high $p_{T}$ region at the LHC. Thus, even if the $v_{2}\left(p_{T}\right)$ curves coincide, the $p_{T}$-integrated $v_{2}$ will be larger at the LHC. The difference in $v_{2}\left(p_{T}\right)$ between the RHIC and the LHC results is that at the LHC the differential $v_{2}$ is more sensitive to the initial profile than at RHIC, i.e. the eBC initialization leads to clearly larger values of $v_{2}\left(p_{T}\right)$ than the eWN initialization, while at RHIC both initializations give very similar results. The reason for this is the larger overall multiplicity and thus longer lifetime of the QGP phase at the LHC. Even in more peripheral collisions the lifetime of the QGP phase is still long enough to generate enough elliptic flow such that the ordering of $v_{2}\left(p_{T}\right)$ between the $\mathrm{eBC}$ and $\mathrm{eWN}$ initialization remains down to $N_{\text {part }} \sim 50$. This can be seen in Fig. 5 .

Although our simple single- $T_{\text {dec }}$ freeze-out model cannot reproduce in detail the measured proton $p_{T}$-spectra and $v_{2}$ at $\mathrm{RHIC}$, it is still interesting to compare the general features in the calculated results for RHIC and the 


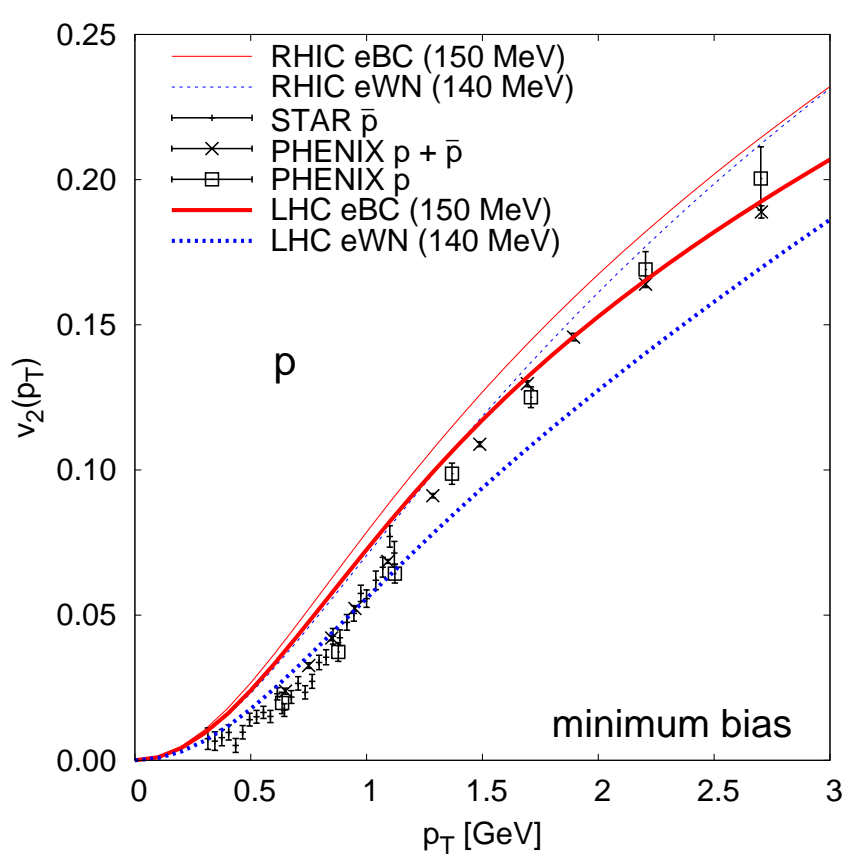

FIG. 8: (Color online) The minimum bias $v_{2}\left(p_{T}\right)$ of protons for $\sqrt{s_{N N}}=200 \mathrm{GeV} \mathrm{Au}+\mathrm{Au}$ collisions at RHIC (thin lines) and $\sqrt{s_{N N}}=5500 \mathrm{GeV} \mathrm{Pb}+\mathrm{Pb}$ collisions at the LHC (thick lines) vs. $p_{T}$. The RHIC data are from the STAR [46] and PHENIX [47, 48] collaborations.

LHC. Figure 8 shows the results from the same calculation as for Fig. 6, but now for protons. Also the RHIC data from STAR [46] and PHENIX [47, 48] collaborations are shown. When the calculations are compared with each other, for both the eBC and eWN initial profiles it is seen that the proton $v_{2}\left(p_{T}\right)$ at fixed $p_{T}$ at the LHC is always below the RHIC values. This can be understood as a mass effect [42]. For the pions, the increase in average $p_{T}$ compensates the increase in the flow asymmetry. However, for protons the increase of average $p_{T}$ is larger due to the larger mass of the proton. Thus the increase of $\left\langle p_{T}\right\rangle$ overcompensates the increase in the flow asymmetry. Although we cannot make a quantitative prediction of $v_{2}\left(p_{T}\right)$ for the protons, we can infer from the results and arguments above that $v_{2}\left(p_{T}\right)$ at the LHC is expected to be below the values measured at RHIC, while, as in the case of pions, the $p_{T}$-integrated $v_{2}$ is still expected to be larger at the LHC than at RHIC.

Finally, we study the uncertainty from multiplicity in predicting the behavior of elliptic flow coefficients from RHIC to the LHC. Motivated by the multiplicity predictions in Refs. 25, 49], we have repeated the calculation for the LHC with half the multiplicity predicted by the EKRT model, by adjusting

$$
\begin{aligned}
\tau_{0} & \rightarrow \sqrt{2} \tau_{0} \\
{[d E / d \eta]_{b=0} } & \rightarrow \frac{1}{2 \sqrt{2}}[d E / d \eta]_{b=0},
\end{aligned}
$$

as suggested by the saturation conjecture 22]. Figure 9 shows the integrated $v_{2}$ for the two different multiplicities at the LHC together with results for RHIC and Fig. 10 the minimum bias $v_{2}\left(p_{T}\right)$. With the lower multiplicity the lifetime of the QGP phase decreases and less elliptic flow is generated, which is clearly seen in Fig. 9, where the integrated $v_{2}$ drops halfway between the RHIC results and the original LHC prediction. The drop is similar between the eBC and eWN initializations. If a low multiplicity is observed at the LHC, the prediction with the eWN profile for $v_{2}$ extends down to similar values as observed at RHIC. The integrated $v_{2}$ is clearly sensitive to the observed multiplicity. The minimum bias $v_{2}\left(p_{T}\right)$, shown in Fig. 10 is not as sensitive to the multiplicity as the integrated elliptic flow, but there is still a visible difference as compared with the high-multiplicity prediction. Already for the high multiplicity calculation with the eWN profile, the LHC prediction is very close to the RHIC data and our calculations for RHIC. When the LHC multiplicity is lowered the eWN result drops at low $p_{T}$ even slightly below the RHIC calculations and data. Also the $\mathrm{eBC}$ prediction drops at low $p_{T}$ closer to the RHIC results.

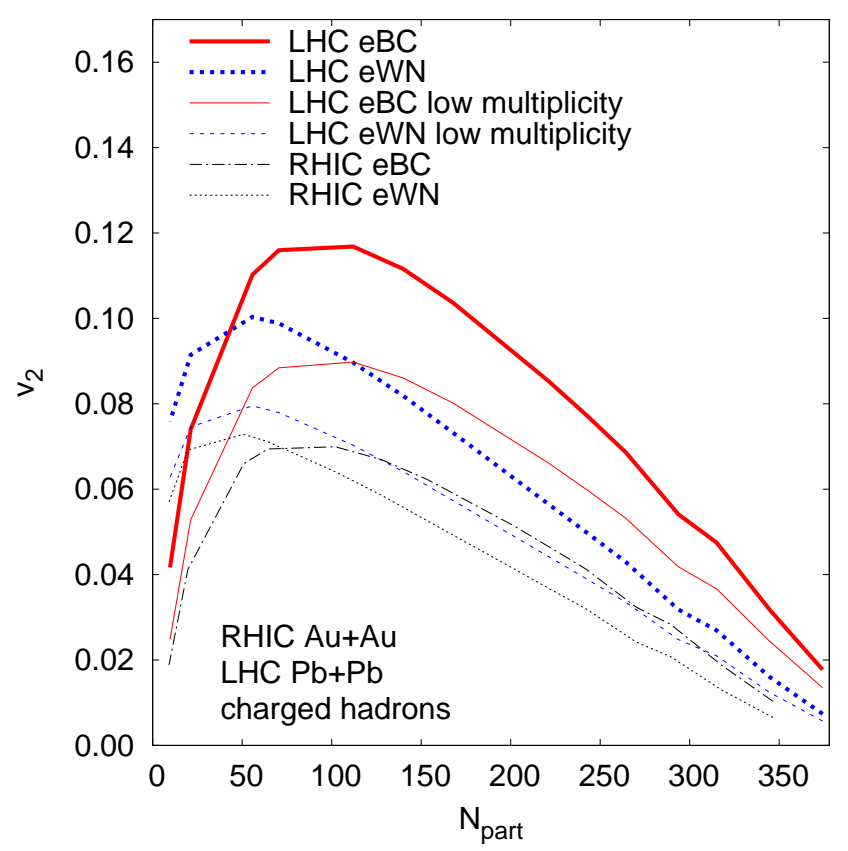

FIG. 9: (Color online) The integrated $v_{2}$ for the LHC lowmultiplicity calculation

\section{CONCLUSIONS}

We study elliptic flow in ultrarelativistic heavy ion collisions at RHIC and LHC energies using the framework of perfect-fluid hydrodynamics. The initial state for central heavy ion collisions is calculated from the pQCD + saturation model [22] and perfect-fluid hydrodynamics is used to model the space-time evolution of the ini- 


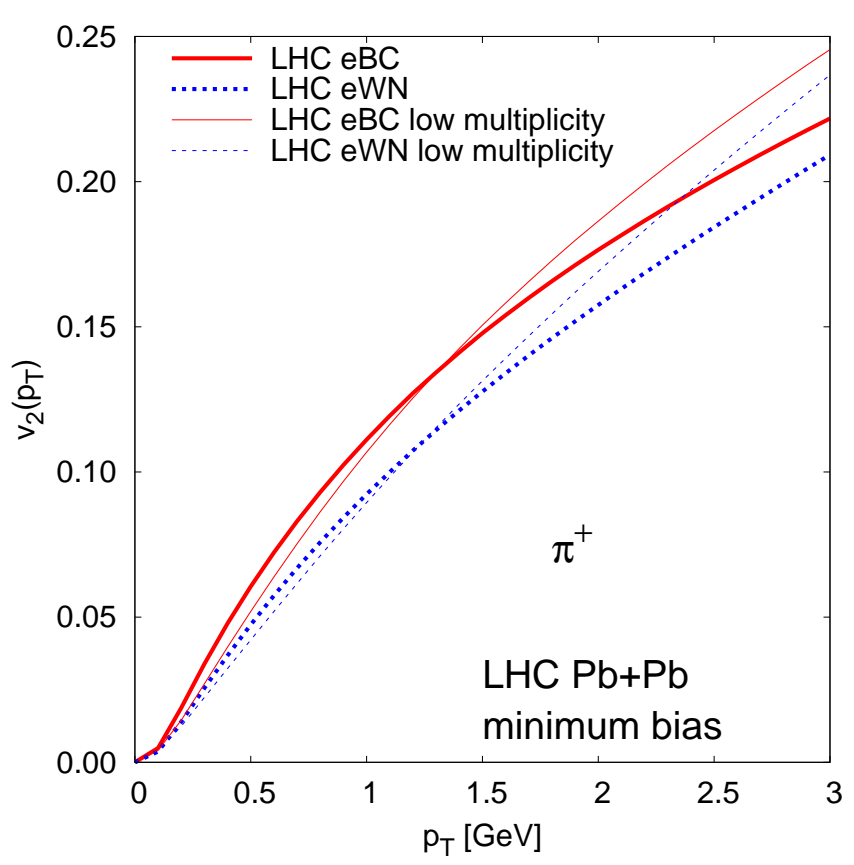

FIG. 10: (Color online) The minimum bias $v_{2}\left(p_{T}\right)$ for the LHC low-multiplicity calculation

tially formed matter. Centrality dependence is modeled by the optical Glauber model. We have shown that the results from this approach are in reasonably good agreement with the measured data at RHIC, and thus provide a good basis to predict both the pion spectra and elliptic flow coefficients in $\sqrt{s_{N N}}=5.5 \mathrm{TeV} \mathrm{Pb}+\mathrm{Pb}$ collisions at the LHC. The main uncertainty in our model is the initial transverse profile of the energy density. This uncertainty is addressed by considering two limits of the Glauber model, taking the energy density proportional either to the density of binary collisions or wounded nucleons. Three main observations are made for the LHC. First we note that the $p_{T}$-integrated $v_{2}$ is expected to be much larger at the LHC than at RHIC, since the lifetime of the QGP phase will be longer and thus the pressure gradients that generate elliptic flow will persist longer. The second observation is that $v_{2}\left(p_{T}\right)$ for pions will be unchanged or may increase slightly from the values measured at RHIC. This can be understood as an interplay between the flow asymmetry and flow magnitude [42]. As the third point we observe that for heavier particles like protons, $v_{2}\left(p_{T}\right)$ will be below the values measured at RHIC, even if the $p_{T}$-integrated $v_{2}$ is larger. This is due to the fact that the increased flow velocity is more important for protons, and thus it overcompensates the increased flow asymmetry.

We further noted that the $p_{T}$ integrated $v_{2}$ has still some sensitivity to the decoupling condition and therefore to the HRG dynamics at RHIC, i.e. that spatial eccentricity is not entirely converted to the momentum space eccentricity before the matter enters the HRG phase. On the contrary, the prediction of $v_{2}$ at the LHC is more robust than at RHIC, once the initial profile and multiplicity are fixed: we do not expect the HRG dynamics to affect significantly the integrated $v_{2}$ at the LHC. The differential elliptic flow coefficient $v_{2}\left(p_{T}\right)$ was in turn found to be insensitive to the decoupling condition at both collision energies. This insensitivity presumably follows from our full equilibrium treatment of the HRG phase. Different HRG dynamics would change this behavior. However, when the decoupling condition is fixed at RHIC to reproduce the hadron spectra, the freeze-out temperature is not expected to change significantly from RHIC to the LHC 37]. Once the EoS and the freeze-out parameters are fixed at RHIC, we expect that the same parameters apply also at the LHC. Once the multiplicities are fixed, the initial transverse profile remains as the largest uncertainty in the model.

The effect of multiplicity on the elliptic flow coefficients at the LHC is studied by considering half the multiplicity that is predicted by the EKRT model. It is found that the integrated $v_{2}$ is quite sensitive to the multiplicity. The lower limit at the LHC, predicted by the eWN initialization, comes even slightly below the upper limit at RHIC given by the eBC initialization. The differential $v_{2}\left(p_{T}\right)$ is found to be less sensitive, but the upper limit given by the eBC model, is moved closer to the RHIC results.

We also note that we expect the applicability region of hydrodynamical modeling to extend higher in $p_{T}$ at the LHC than at RHIC, because pions from jet fragmentation should start to dominate particle production over the hydrodynamical spectra at higher $p_{T}$ at the LHC [20]. Therefore we expect that the minimum bias $v_{2}\left(p_{T}\right)$ will reach higher values at the LHC even if the low- $p_{T}$ results are similar to those measured at RHIC.

\section{Acknowledgments}

We thank the Academy of Finland, the project 115262, for financial support and P. Huovinen for discussions.
[1] J. Y. Ollitrault, Phys. Rev. D 46, 229 (1992).

[2] P. Kolb and U. W. Heinz, in Quark-Gluon Plasma 3, edited by R. C. Hwa and X. N. Wang, (World Scientific, Singapore, 2004), arXiv:nucl-th/0305084

[3] P. Huovinen, in Quark-Gluon Plasma 3, edited by
R. C. Hwa and X. N. Wang, (World Scientific, Singapore, 2004), arXiv:nucl-th/0305064.

[4] P. Huovinen and P. V. Ruuskanen, Ann. Rev. Nucl. Part. Sci. 56, 163 (2006) arXiv:nucl-th/0605008.

[5] F. Cooper and G. Frye, Phys. Rev. D 10, 186 (1974). 
[6] M. Gyulassy and L. McLerran, Nucl. Phys. A 750, 30 (2005) arXiv:nucl-th/0405013.

[7] P. F. Kolb, J. Sollfrank and U. W. Heinz, Phys. Rev. C 62, 054909 (2000) arXiv:hep-ph/0006129.

[8] T. Hirano and K. Tsuda, Phys. Rev. C 66, 054905 (2002) arXiv:nucl-th/0205043.

[9] P. F. Kolb and R. Rapp, Phys. Rev. C 67, 044903 (2003) arXiv:hep-ph/0210222.

[10] P. Huovinen, arXiv:0710.4379 [nucl-th].

[11] D. Teaney, J. Lauret and E. V. Shuryak, arXiv:nucl-th/0110037

[12] T. Hirano, U. W. Heinz, D. Kharzeev, R. Lacey and Y. Nara, Phys. Rev. C 77, 044909 (2008) arXiv:0710.5795 [nucl-th]].

[13] T. Hirano, U. W. Heinz, D. Kharzeev, R. Lacey and Y. Nara, Phys. Lett. B 636, 299 (2006) arXiv:nucl-th/0511046.

[14] C. Nonaka and S. A. Bass, Phys. Rev. C 75, 014902 (2007) arXiv:nucl-th/0607018.

[15] P. Romatschke and U. Romatschke, Phys. Rev. Lett. 99, 172301 (2007) arXiv:0706.1522 [nucl-th]].

[16] H. Song and U. W. Heinz, arXiv:0712.3715 [nucl-th].

[17] M. Luzum and P. Romatschke, arXiv:0804.4015 [nucl-th].

[18] K. Dusling and D. Teaney, Phys. Rev. C 77, 034905 (2008) arXiv:0710.5932 [nucl-th]].

[19] A. K. Chaudhuri, arXiv:0801.3180 [nucl-th].

[20] K. J. Eskola, H. Honkanen, H. Niemi, P. V. Ruuskanen and S. S. Räsänen, Phys. Rev. C 72, 044904 (2005) arXiv:hep-ph/0506049.

[21] K. J. Eskola, H. Niemi, P. V. Ruuskanen and S. S. Räsänen, Phys. Lett. B 566, 187 (2003) arXiv:hep-ph/0206230.

[22] K. J. Eskola, K. Kajantie, P. V. Ruuskanen and K. Tuominen, Nucl. Phys. B 570, 379 (2000) arXiv:hep-ph/9909456.

[23] K. J. Eskola, P. V. Ruuskanen, S. S. Räsänen and K. Tuominen, Nucl. Phys. A 696 (2001) 715 arXiv:hep-ph/0104010.

[24] K. J. Eskola, H. Niemi and P. V. Ruuskanen, arXiv:0705.2114 [hep-ph].

[25] N. Armesto et al., J. Phys. G 35, 054001 (2008) arXiv:0711.0974 [hep-ph]].

[26] T. Hirano, Prog. Theor. Phys. Suppl. 168, 347 (2007) arXiv:0704.1699 [nucl-th]].

[27] E. Iancu and R. Venugopalan, in Quark-Gluon Plasma 3, edited by R. C. Hwa and X. N. Wang, (World Scientific,
Singapore, 2004), arXiv:hep-ph/0303204

[28] M. Bluhm, B. Kampfer, R. Schulze, D. Seipt and U. Heinz, Phys. Rev. C 76, 034901 (2007) arXiv:0705.0397 [hep-ph]].

[29] M. Chojnacki, W. Florkowski, W. Broniowski and A. Kisiel, arXiv:0712.0947 [nucl-th].

[30] A. K. Chaudhuri, arXiv:0803.0643 [nucl-th].

[31] J. D. Bjorken, Phys. Rev. D 27, 140 (1983).

[32] J.P. Boris and D.L. Book, J. Comp. Phys. A11, (1973) 38; D.L. Book, J.P. Boris and K. Hain, J. Comp. Phys. A18, (1975) 248

[33] S.T. Zalesak, J. Comp. Phys. A31, (1979) 335

[34] J. Sollfrank, P. Huovinen, M. Kataja, P. V. Ruuskanen, M. Prakash and R. Venugopalan, Phys. Rev. C 55, 392 (1997) arXiv:nucl-th/9607029.

[35] D. E. Groom et al. [Particle Data Group], Eur. Phys. J. C 15 (2000) 1.

[36] P. Huovinen, Nucl. Phys. A 761, 296 (2005) arXiv:nucl-th/0505036.

[37] K. J. Eskola, H. Niemi and P. V. Ruuskanen, Phys. Rev. C 77, 044907 (2008) arXiv:0710.4476 [hep-ph]].

[38] U. Heinz and G. Kestin, PoS CPOD2006, 038 (2006) arXiv:nucl-th/0612105.

[39] K. J. Eskola, K. Kajantie and K. Tuominen, Phys. Lett. B 497, 39 (2001) arXiv:hep-ph/0009246.

[40] K. J. Eskola, K. Kajantie and K. Tuominen, Nucl. Phys. A 700, 509 (2002) arXiv:hep-ph/0106330.

[41] P. F. Kolb, U. W. Heinz, P. Huovinen, K. J. Eskola and K. Tuominen, Nucl. Phys. A 696, 197 (2001) arXiv:hep-ph/0103234.

[42] P. Huovinen, P. F. Kolb, U. W. Heinz, P. V. Ruuskanen and S. A. Voloshin, Phys. Lett. B 503, 58 (2001) arXiv:hep-ph/0101136.

[43] S. S. Adler et al. [PHENIX Collaboration], Phys. Rev. C 69, 034909 (2004) arXiv:nucl-ex/0307022.

[44] B. Alver et al. [PHOBOS Collaboration], Phys. Rev. Lett. 98, 242302 (2007) arXiv:nucl-ex/0610037.

[45] M. Miller and R. Snellings, arXiv:nucl-ex/0312008.

[46] J. Adams et al. [STAR Collaboration], Phys. Rev. C 72, 014904 (2005) arXiv:nucl-ex/0409033.

[47] A. Adare et al. [PHENIX Collaboration], Phys. Rev. Lett. 98, 162301 (2007) arXiv:nucl-ex/0608033.

[48] S. S. Adler et al. [PHENIX Collaboration], Phys. Rev. Lett. 91, 182301 (2003) arXiv:nucl-ex/0305013.

[49] N. Armesto, arXiv:0804.4158 [hep-ph]. 